\title{
Emergent spontaneous symmetry breaking and emergent symmetry restoration in rippling gravitational background
}

\author{
Maxim A. Kurkov ${ }^{\mathrm{a}}$ \\ CMCC, Universidade Federal do ABC, Santo André, SP, Brazil
}

Received: 19 March 2016 / Accepted: 2 June 2016 / Published online: 16 June 2016

(c) The Author(s) 2016. This article is published with open access at Springerlink.com

\begin{abstract}
We study effects of a rippling gravitational background on a scalar field with a double well potential, focusing on the analogy with the well known dynamics of the Kapitza's pendulum. The ripples are rendered as infinitesimal but rapidly oscillating perturbations of the scale factor. We find that the resulting dynamics crucially depends on a value of the parameter $\xi$ in the $\xi R \phi^{2}$ vertex. For the time-dependent perturbations of a proper form the resulting effective action is generally covariant, and at a high enough frequency at $\xi<0$ and at $\xi>1 / 6$ the effective potential has a single minimum at zero, thereby restoring spontaneously broken symmetry of the ground state. On the other side, at $0<\xi<1 / 6$ spontaneous symmetry breaking emerges even when it is absent in the unperturbed case.
\end{abstract}

\section{Introduction}

Oscillating gravitational backgrounds attract attention of theoretical physicists in various contexts starting from quantum decoherence [1,2] and finishing some cosmological [3] and extra dimensional constructions [4-6]. Effects of a rippling scale factor on a minimally coupled quantized scalar field were studied in [7]. One can also find some interesting consequences of rippling parameters in $[8,9]$. The first famous example of a rippling background, however, is not related to quantum physics or cosmology - it is the famous Kapitza's pendulum $[10,11]$, i.e. a classical pendulum whose suspension point rapidly oscillates with a small amplitude. The highly nontrivial and counterintuitive classical dynamics of this simple object inspired us to write this article. As we show below already at classical level small but rapidly oscillating perturbations of the scale factor may affect the ground states of physically relevant models in a quite interesting way.

In this paper we study the effect of the rapidly oscillating gravitational field $g_{\mu \nu}$ on a classical dynamics of the scalar

a e-mail: max.kurkov@gmail.com field $\phi$. The latter is described by the most general action ${ }^{1}$

$S_{g}[\phi]=\int \mathrm{d}^{4} x \sqrt{-g}\left\{\frac{1}{2} \phi\left(-\nabla_{g}^{2}-\xi R_{g}+\mu^{2}\right) \phi-\frac{1}{4 !} \lambda \phi^{4}\right\}$,

which leads to renormalizable ${ }^{2}$ quantum field theory in four dimensions, which is invariant upon the reflection $\phi \longrightarrow$ $-\phi$. Such an action is of special importance, since the Higgs field of the Standard Model is described by the action of such a form. We consider the metric tensor given by the product of the slowly varying bare ${ }^{3}$ metric $\bar{g}_{\mu \nu}$ and the highly oscillating conformal factor, which is very close to one:

$g_{\mu \nu}=\bar{g}_{\mu \nu} \mathrm{e}^{2 \alpha \cos (\omega t)}$

where the dimensionless amplitude $\alpha$ is much smaller than 1 , the frequency $\omega$ is much larger than $\mu$ and invariants of the metric tensor $\bar{g}_{\mu \nu}$ (and its derivatives) of the dimension [length ${ }^{-1}$ ]. The main goal of the present paper is to generalize the result of Kapitza to this model. In particular, we show that solutions of the equation of motion, which comes out from the action (1), split into two parts

$\phi=\bar{\phi}+\delta \phi$,

where $\delta \phi$ is a rapidly oscillating and small correction, which approaches to zero as $\omega^{-1}$ in the high frequency limit, while the dynamics of the slowly varying field $\bar{\phi}$ is described by the classical action, which we establish. Surely due to a manifest frame dependence of the perturbation law (2), the effective

\footnotetext{
${ }^{1}$ Hereafter the lower index specifies which metric tensor is used in order to construct a given quantity.

2 This statement refers to quantum field theory on a classical gravitational background.

${ }^{3}$ In what follows the word "bare" is addressed to the unperturbed theory i.e. at $\omega=0$.
} 
action generally speaking depends on the choice of the coordinates, which are used to perturb the bare metric tensor $\bar{g}_{\mu \nu}$.

The most interesting result is related to the situation, when the oscillating metric takes the simple form (2) in the comoving frame. ${ }^{4}$ In such a case the effective action has exactly the same structure (1), where the quantities $g_{\mu \nu}$ and $\phi$ are replaced correspondingly by $\bar{g}_{\mu \nu}$ and $\bar{\phi}$,

$S_{\bar{g}}^{\mathrm{eff}}[\bar{\phi}]=\int \mathrm{d}^{4} x \sqrt{-\bar{g}}\left\{\frac{1}{2} \bar{\phi}\left(-\nabla_{\bar{g}}^{2}-\xi R_{\bar{g}}+\mu_{\mathrm{eff}}^{2}\right) \bar{\phi}-\frac{1}{4 !} \lambda \bar{\phi}^{4}\right\}$,

therefore it is generally covariant, and the only effect of the oscillations is an additive renormalization of the mass parameter $\mu^{2}$. A sign of the correction $\Delta \mu^{2} \equiv \mu_{\text {eff }}^{2}-\mu^{2}$ nontrivially depends on a choice of the coupling constant $\xi$, and this is probably the most interesting result of the paper.

We find that at $\xi<0$ and at $\xi>1 / 6$ the correction $\Delta \mu^{2}$ is always negative, and at the high enough frequency $\omega$ it can easily compensate the bare mass parameter, what quite naturally implies that the effect of the rapid oscillations is similar to an increase in temperature: the effective potential (in contrast to the original one) has just a single minimum at $\phi=0$, and spontaneous symmetry breaking of the bare theory vanishes. All particles which obtain their mass due to the Higgs mechanism become massless.

In the another regime $0<\xi<1 / 6$ the situation changes qualitatively. The correction $\Delta \mu^{2} \equiv \mu_{\text {eff }}^{2}-\mu^{2}$ becomes positive, and even when the mass parameter is absent in the bare theory, ${ }^{5}$ it appears in the effective one. Such an effect can be regarded as some sort of emergent spontaneous symmetry breaking. At $\xi=1 / 6$ (naturally) and $\xi=0$ (less naturally) the effect is absent.

An influence of time dependent (but not oscillating) gravitational backgrounds on the vacuum state of a scalar field was studied in the context of inflation in [12], and the result crucially depends on the non minimal coupling $\xi$ as well, in particular at $\xi=1 / 6$ the influence is minimal. Nevertheless, the effects discussed in [12] and here are different, since in contrast to our setup in the framework of [12] at $\xi=0$ the vacuum expectation value grows up exponentially with time.

This paper is organized as follows. In Sect. 2 we briefly describe relevant aspects of the dynamics of the Kapitza's pendulum in order to apply the same technique in the forthcoming discussion. In Sect. 3 we derive the effective action (1) and establish the renormalized mass parameter $\mu_{\mathrm{eff}}^{2}$. Section 4 is devoted to numerical illustrations of the results obtained for the flat bare metric $\bar{g}_{\mu \nu}$.

\footnotetext{
${ }^{4}$ Actually the result holds for a bigger class of perturbations, see the discussion in Sect. 3.

5 Or it is negative.
}

\section{Kapitza's pendulum: a brief technical review}

The mathematical pendulum is described by the following equation of motion

$\ddot{f}+g \sin (f)=\ddot{f}+g f+\mathcal{O}\left(f^{3}\right)=0$,

where $f(t)$ is the angular coordinate (we consider the length to be equal to one), and $g>0$ is the free-fall acceleration; hereafter the dot stands for differentiation with respect to time. The only stable equilibrium position is located at $f=0$, but the situation changes dramatically, when the pivot vibrates with the high frequency $\omega$. In such a case the equation of motion reads

$\ddot{f}+\left(g+\alpha \omega^{2} \cos (\omega t)\right) \sin (f)=0$,

where the constant $\alpha$ stands for the amplitude of the oscillations of the suspension, and it is supposed to be small. The most amazing fact is the appearance of a new stable equilibrium point in the inverted position i.e. at $f=\pi$. More precisely the solution of the equation of motion is given by a sum of the two functions $\bar{f}(t)$ and $\delta f(t)$, where the former satisfies some "effective" equation of motion, which in particular exhibits slow oscillations in the vicinity of $f=\pi$, when the frequency $\omega$ is high enough. The latter is a rapidly oscillating correction of the order ${ }^{6}$ of $\mathcal{O}\left(\omega^{-1}\right)$. It is remarkable that one can relatively easily give a precise mathematical description of such a nontrivial and unexpected phenomenon. Below we present a derivation of this well known result in the form which is the most suitable and clear for the forthcoming analysis of the scalar field in a rapidly oscillating gravitational background.

\section{Step 1. Proper ansatz}

A numerical analysis of the Eq. (6) hints us, that its solution splits into the slowly varying part $\bar{f}(t)$ and the rapidly oscillating but small correction $\delta f$. It is natural to expect that the correction $\delta f$ oscillates with the same frequency $\omega$, therefore let us look for the solution in a form of an asymptotic expansion in inverse powers of $\omega$

$$
f=\bar{f}+\frac{1}{\omega}(A \sin (\omega t)+B \cos (\omega t))+\mathcal{O}\left(\frac{1}{\omega^{2}}\right),
$$

where the (yet undetermined) functions $A(t)$ and $B(t)$ vary slowly. ${ }^{7}$ Substituting the ansatz (7) and $\alpha=\gamma \cdot \omega^{-1}$ in the equation of motion (6) we obtain at the leading order at large $\omega$ :

\footnotetext{
${ }^{6}$ Actually this asymptotic takes place in the double limit $\alpha \rightarrow 0$, and $\omega \rightarrow \infty$, while their product $\alpha \cdot \omega \equiv \gamma$ is a constant.

7 At this point this is just an assumption to be checked a posteriori, after all the functions are found.
} 


$$
\begin{aligned}
0= & \mathcal{F}(t)=\omega\{\sin (\bar{f}(t)) \gamma \cos (\omega t) \\
& -B(t) \cos (\omega t)-A(t) \sin (\omega t)\}+\mathcal{O}\left(\omega^{0}\right),
\end{aligned}
$$

what defines $A(t)=0$ and $B(t)=\gamma \sin (\bar{f})$. From this moment on the ansatz (7) contains just one undetermined function $\bar{f}$.

\section{Step 2. Averaging over rapid oscillations}

Substituting $A(t)=0$ and $B(t)=\gamma \sin (\bar{f})$ in the next to the leading order of our asymptotic expansion (8) we immediately arrive to the equation which determines $\bar{f}(t)$.

$$
\begin{aligned}
0= & \mathcal{F}(t)=\ddot{\bar{f}}+\sin (\bar{f}) g-2 \cos (\bar{f}) \dot{\bar{f}} \gamma \sin (\omega t) \\
& +\cos (\bar{f}) \gamma^{2}(\cos (\omega t))^{2} \sin (\bar{f})+\mathcal{O}\left(\omega^{-1}\right)
\end{aligned}
$$

Now let us average the function $\mathcal{F}(t)$ over the period of rapid oscillations $\frac{2 \pi}{\omega}$ :

$\langle\mathcal{F}\rangle(t) \equiv \frac{\omega}{2 \pi} \int_{0}^{\frac{2 \pi}{\omega}} \mathrm{d} \tau \mathcal{F}(\tau+t)$.

Since by our assumption the quantity $\bar{f}$ and its derivative vary slowly during the period of rapid oscillations, one can easily compute the integral (10) in the limit $\omega \rightarrow \infty$, and the answer reads:

$$
\ddot{\bar{f}}+\sin (\bar{f}) g+\frac{1}{2} \cos (\bar{f}) \gamma^{2} \sin (\bar{f})=0, \quad \gamma \equiv \omega \cdot \alpha \ll \omega .
$$

The Eq. (11) is autonomous and it does not contain large parameters anymore, therefore $\bar{f}$ is indeed slowly varying function, ${ }^{8}$ what justifies all our assumptions.

Small deviations of $\bar{f}$ from zero satisfy the standard pendulum Eq. (5) where $g$ is replaced by $g_{\text {eff }}$

$g_{\text {eff }}^{\text {down }}=g+\frac{1}{2} \alpha^{2} \omega^{2}$,

thus in the vicinity of this equilibrium point one has an additive (finite) renormalization of the free fall acceleration constant $g$. On the other side, and this is even more interesting, small deviations from $\bar{f}=\pi$ satisfy linearized version of Eq. (5) with

$g_{\text {eff }}^{\text {up }}=-g+\frac{1}{2} \alpha^{2} \omega^{2}>0, \quad$ at $|\omega \cdot \alpha|>\sqrt{2 g}$,

therefore in this regime the pendulum starts to oscillate near its upper position with the slow frequency $\Omega \equiv$ $\sqrt{-g+\frac{1}{2} \alpha^{2} \omega^{2}} \ll \omega$. Below we consider the system described

\footnotetext{
${ }^{8}$ Unless one chooses large initial velocity.
}

by the action (1) along similar lines, and we find that it shares some similarities with the Kapitza's pendulum.

\section{Scalar field in oscillating gravitational background}

Now we study the effect of the rapid oscillations defined by (2) on the dynamics of the scalar field $\phi$, which obeys the classical equation of motion

$$
-\frac{1}{\sqrt{-g}} \frac{\delta S_{g}[\phi]}{\delta \phi}=\left(\nabla_{g}^{2}+\xi R_{g}-\mu^{2}\right) \phi+\frac{1}{3 !} \lambda \phi^{3}=0 .
$$

The gravitational background is considered to be given, and we assume that the back-reaction of the scalar field on its dynamics is negligibly small. We simplify the forthcoming analysis substituting

$\phi=\mathrm{e}^{-\alpha \cos (\omega t)} \tilde{\phi}$

On the one hand, due to the local Weyl invariance of the action (1) at $\xi=\frac{1}{6}$ the Eq. (14) rewritten in terms of $\tilde{\phi}$ has simpler dependence on $\alpha$ and $\omega$. On the other hand, we are interested in the evolution of the slowly varying part of $\phi$, which coincides for $\tilde{\phi}$ and $\phi$.

In order to carry out the computations it makes sense to rewrite the equation of motion splitting explicitly rapidly and slowly varying quontities

$\left(\bar{g}^{\mu \nu} \partial_{\mu \nu}^{2}+Y^{\mu} \partial_{\mu}+\xi R_{\bar{g}}-\mu^{2}\right) \tilde{\phi}+\frac{\lambda}{3 !} \tilde{\phi}^{3}+f_{\alpha}=0$

where $\bar{g}^{\mu \nu}, Y^{\mu} \equiv \frac{1}{\sqrt{-\bar{g}}}\left(\partial_{\nu} \bar{g}^{\mu \nu} \sqrt{-\bar{g}}\right)$ and $R_{\bar{g}}$ vary slowly by construction. The function $f_{\alpha}$ absorbs all influence of the rapidly oscillating exponential factor in (2), and it is defined by

$$
\begin{aligned}
f_{\alpha} \equiv & {\left[( 6 \xi - 1 ) \left(-\omega^{2} \alpha \bar{g}^{00}\left(\cos (\omega t)-\alpha \sin ^{2}(\omega t)\right)\right.\right.} \\
& \left.\left.-\omega \alpha Y^{0} \sin (\omega t)\right)+\mu^{2}\left(1-\mathrm{e}^{2 \alpha \cos (\omega t)}\right)\right] \tilde{\phi}
\end{aligned}
$$

Below we study the leading asymptotic at large $\omega$ and small $\alpha$ keeping $\alpha=\gamma / \omega=\mathcal{O}\left(\omega^{-1}\right)$ by analogy with the Kapitza's pendulum.

\section{Step 1. Proper ansatz}

Inspired by the example discussed in the previous section we look for the solution in a form of the following asymptotic ansatz:

$\tilde{\phi}=\bar{\phi}+\frac{1}{\omega}(T \cos (\omega t)+W \sin (\omega t))+\mathcal{O}\left(\frac{1}{\omega^{2}}\right)$,

where $T$ and $W$ are slowly varying functions of all coordinates. We do not write the tilde over $\bar{\phi}$, since, as we said 
above, in the limit of infinitely large $\omega$ (and infinitely small $\alpha$ ) the quantities $\phi$ and $\tilde{\phi}$ coincide. Substituting the ansatz (18) in the equation of motion (16) we immediately determine $T$ and $W$ :

$$
\begin{aligned}
0=\mathcal{F} \equiv & \omega\left(-6 \xi \gamma \bar{g}^{00} \cos (\omega t) \bar{\phi}-\bar{g}^{00} T \cos (\omega t)\right. \\
& \left.-\bar{g}^{00} W \sin (\omega t)+\gamma \bar{g}^{00} \cos (\omega t) \bar{\phi}\right)+\mathcal{O}\left(\omega^{0}\right),
\end{aligned}
$$

what implies

$T=(1-6 \xi) \gamma \bar{\phi}, \quad W=0$.

As in the "canonical" example there is just one undetermined function $\bar{\phi}$.

\section{Step 2. Averaging over rapid oscillations}

Exactly as we did in the previous section, substituting (20) in the next to the leading order of our asymptotic expansion (19) we derive the equation which determines $\bar{\phi}$.

$$
\begin{aligned}
0= & \mathcal{F} \equiv\left(\bar{g}^{\mu \nu} \partial_{\mu \nu}^{2}+Y^{\mu} \partial_{\mu}+\xi R_{\bar{g}}-\mu^{2}\right) \bar{\phi}+\frac{\lambda}{3 !} \bar{\phi}^{3} \\
& +(6 \xi-1)\left[2 \gamma \bar{g}^{\mu 0}\left(\partial_{\mu} \bar{\phi}\right) \sin (\omega t)\right. \\
& \left.+\gamma^{2} \bar{g}^{00} \bar{\phi}\left(6(\cos (\omega t))^{2} \xi-\cos (2 \omega t)\right)\right]+\mathcal{O}\left(\frac{1}{\omega}\right)
\end{aligned}
$$

Averaging over the period of rapid oscillations (c.f. Eq. (10)) and passing to the limit of large $\omega$ we obtain the final effective equation for $\bar{\phi}$ :

$$
\begin{aligned}
0= & \lim _{\omega \rightarrow \infty}\langle\mathcal{F}\rangle=\left(\nabla_{\bar{g}}^{2}+\xi R_{\bar{g}}-\mu^{2}+3 \gamma^{2} \bar{g}^{00} \xi(6 \xi-1)\right) \bar{\phi} \\
& +\frac{1}{3 !} \lambda \bar{\phi}^{3} .
\end{aligned}
$$

The structure of Eq. (22) immediately suggests us, which oscillations of the form (2) are the most interesting. Surely we wish to obtain a generally covariant expression by the end of the day, what happens if and only if the simple perturbation equation (2) is written in the frame where $\bar{g}^{00}$ does not depend on coordinates e.g. the comoving frame. We emphasize that we are talking about preferred ways of perturbation of the bare metric $\bar{g}_{\mu \nu}$ rather than preferred coordinate systems. Starting from now we assume that the perturbations are "good" in the mentioned above sense, and ${ }^{9}$ the coordinates in Eq. (2) are chosen in such a way that $\bar{g}^{00}=+1$, so we arrive to the manifestly generally covariant equation

\footnotetext{
${ }^{9}$ In principle $\bar{g}^{00}$ must not be necessary positive and it can be either negative or zero (e.g. the light front coordinates). In the former case the effect changes its sign, and in the latter case it is absent.
}

$0=\left(\nabla_{\bar{g}}^{2}+\xi R_{\bar{g}}-\mu_{\text {eff }}^{2}\right) \bar{\phi}+\frac{1}{3 !} \lambda \bar{\phi}^{3}=-\frac{1}{\sqrt{-\bar{g}}} \frac{\delta S_{\bar{g}}^{\text {eff }}[\bar{\phi}]}{\delta \bar{\phi}}$,

which holds for an arbitrary coordinate system, ${ }^{10}$ and has exactly the same structure as Eq. (14). The effective mass parameter is given by

$\mu_{\text {eff }}^{2}=\mu^{2}+3 \alpha^{2} \omega^{2} \xi(1-6 \xi) \ll \omega^{2}$.

As we announced in the Sect. 1, this quantity can be either positive or negative depending on the ratios between the parameters, what may dramatically affect the spontaneous symmetry breaking. The particular form (24) of the additive renormalization of the mass parameter $\mu^{2}$ suggests us to consider two different regimes of the non minimal scalar-tensor coupling, defined by the dimensionless coupling constant $\xi$.

- At $\xi<0$ and $\xi>1 / 6$ the correction to the mass parameter is negative, and at

$$
|\omega \cdot \alpha|>\frac{\mu}{\sqrt{3 \xi(6 \xi-1)}}, \quad \mu^{2}>0,
$$

the effective mass parameter becomes negative, what restores a spontaneously broken symmetry of the ground state. This effect is similar to the dynamical stabilization of the upper equilibrium position of the Kapitza's pendulum, c.f. Eq. (13).

- At $0<\xi<1 / 6$ the correction is positive, what is similar to the effective renormalization of the free fall acceleration in the context of the Kapitza's pendulum oscillating near its lower equilibrium, c.f. (12). This regime becomes more interesting when the "bare theory" is not spontaneously broken, i.e. at $\mu^{2}=-m^{2}<0$. In such a situation at

$|\omega \cdot \alpha|>\frac{m}{\sqrt{3 \xi(1-6 \xi)}}, \quad m^{2} \equiv-\mu^{2}>0$,

the effective mass parameter $\mu_{\text {eff }}^{2}$ becomes positive in contrast to the bare one $\mu^{2}$, what implies emergent spontaneous symmetry breaking. One can easily see, that this effect reaches its maximum at $\xi=1 / 12$.

If for some application one prefers to avoid the mentioned above effects, one has to work with the minimal $\xi=0$ or the conformal $\xi=1 / 6$ couplings.

Remark We notice that the coordinate $x_{0} \equiv t$ must not be necessary the conformal time, but it can be also the comoving time $t_{c}$. Let us fix the gauge by the following condition

\footnotetext{
$\overline{10}$ Surely, for an arbitrary frame one has to rewrite Eq. (2), which defines the perturbations, according to the tensor transformation law.
} 
$\bar{g}_{00}=1, \quad \bar{g}_{0 j}=0, \quad j=1,2,3$,

so the square of the infinitesimal four interval reads (c.f. Eq. (2)):

$\mathrm{d} s^{2}=\left(\mathrm{d} t^{2}-\mathrm{d} \mathbf{x}^{2}\right) \mathrm{e}^{2 \alpha \cos (\omega t)}=\mathrm{d} t_{c}^{2}-\left(\mathrm{d} \mathbf{x}^{2}\right) \mathrm{e}^{2 \alpha \cos \left(\omega t_{c}\right)}$,

where $\mathrm{d} \mathbf{x}^{2} \equiv \bar{g}_{i j} \mathrm{~d} x^{i} \mathrm{~d} x^{j}, i, j=1,2,3$. In other words instead of consideration of the fluctuations of the form (2) we could have considered

$g_{00}=\bar{g}_{00}, \quad g_{i j}=\bar{g}_{i j} \mathrm{e}^{2 \alpha \cos (\omega t)}, \quad i, j=1,2,3$

from the very beginning, ${ }^{11}$ what is more natural for Friedmann-Lemaittre-Robertson-Walker metric. All the results viz the final effective equation (23), the renormalization of the mass parameter (24) and the conditions (25), (26), which define the phase transitions, remain absolutely the same. Note that in (29) we wrote $t$ instead of $t_{c}$, since up to a correction of the order of $\mathcal{O}\left(\omega^{-1}\right)$ these two quantities (but not their derivatives!) coincide:

$t=\int_{0}^{t_{c}} \mathrm{~d} z \mathrm{e}^{-2 \alpha \cos (\omega z)}=t_{c}+\mathcal{O}\left(\omega^{-1}\right), \quad \alpha \equiv \frac{\gamma}{\omega} \ll 1$.

So far we discussed the ripples along the time direction $t \equiv x^{0}$. One can easily elaborate the oscillations along some spatial direction e.g. $z \equiv x^{3}$ in a similar manner. Replacing $t$ by $z$ in the oscillating conformal factor in (2) and repeating all the discussion we arrive to (22), where $\bar{g}^{00}$ is replaced by $\bar{g}^{33}$. The effective equation becomes generally covariant if and only if the (modified) perturbation law (2) is written in the coordinate system, where $\bar{g}^{33}=$ const, for example in the Gaussian normal coordinates in respect to the hypersurface of constant $z$. It is worth noting that for such a choice of coordinates in the perturbation law the effect of this "spatial" rippling has the opposite sign with respect to the timedependent rippling introduced in the comoving frame, since $\bar{g}^{33}=-1$ in the Gaussian normal coordinates.

In the next section we illustrate numerically how this mechanism works on a few examples.

\section{Numerical illustrations}

As we have seen in the previous section the behavior of the scalar field in a rapidly oscillating gravitational background crucially depends on two entries: the parameter $\gamma \equiv \alpha \cdot \omega$, which comprises the information on the perturbation, and

\footnotetext{
${ }^{11}$ Upon the condition (27).
}

the parameter $\xi$, which is responsible for the non minimal tensor-scalar coupling.

Below we solve numerically the exact (14) and the effective (23) equations of motion for various values of the parameters $\gamma$ and $\xi$. The slowly varying background metric is chosen to be flat $\bar{g}_{\mu \nu}=\eta_{\mu \nu}$, and the perturbations are taken of the form (29). Since the metric $g_{\mu \nu}$ depends just on time, for the sake of simplicity we consider the field $\phi$ to be independent on the spatial coordinates, i.e. $\phi=\phi(t)$. In such a setup the exact and the effective equations correspondingly read:

$$
\begin{gathered}
\ddot{\phi}-3 \gamma \sin (\omega t) \dot{\phi}+\left(-6 \xi \omega \gamma \cos (\omega t)+6 \xi \gamma^{2}\right. \\
\left.-6 \xi \gamma^{2} \cos (2 \omega t)-\mu^{2}\right) \phi+\frac{1}{3 !} \lambda \phi^{3}=0
\end{gathered}
$$

and

$\ddot{\phi}-\underbrace{\left(\mu^{2}+3 \gamma^{2} \xi(1-6 \xi)\right)}_{\mu_{\mathrm{eff}}^{2}} \phi+\frac{1}{3 !} \lambda \phi^{3}=0$,

where $\gamma \equiv \alpha \cdot \omega$. On the figures Figs. 1, 2, 3 and 4 numerical solutions of the exact and the effective equations are undistinguishable in agreement with the fact that the difference between the exact and the effective solutions is of the order of $\omega^{-1} \sim 10^{-3}$ for our choice of $\omega$. For a short distance behavior see Fig. 5. Throughout this section ${ }^{12} \gamma_{0} \equiv \frac{|\mu|}{\sqrt{|3 \xi(6 \xi-1)|}}$ stands for the critical value of the parameter $\gamma$.

Let us consider the system which is spontaneously bro$\operatorname{ken}^{13}$ at $\omega=0$. Varying the parameter $\gamma \equiv \alpha \cdot \omega$ above and below its critical value we solve numerically the Eqs. (31), (32); corresponding solutions are presented on Fig. 1. As one would have expected for small negative deviations $\Delta \gamma$ of $\gamma$ from the critical value $\gamma_{0}$ the effect of rapid oscillations results in a shift of the frequency of slow oscillations and a shift of the (nonzero) equilibrium point. When $\Delta \gamma \geq 0$ the solutions with high precision are described by slow oscillations around the symmetric ground state $\phi=0$, so effectively at the scales of length much higher than $\omega^{-1}$ the system behaves as non spontaneously broken.

Corresponding dependence on the non minimal scalartensor coupling $\xi$ is presented on Fig. 2. Inside the interval $0<\xi<1 / 6$ the only effect is a shift of the frequency of small oscillations around the shifted equilibrium point $\phi \neq 0$, see Fig. 2c and d. At $\xi=0$ and $\xi=\frac{1}{6}$ the effect is absent: the bare $(\omega=0)$ and the perturbed curves (up to small deviations $\sim \omega^{-1}$ ) coincide, Fig. $2 \mathrm{~b}$ and e. Finally at $\xi<0$ and at $\xi>\frac{1}{6}$ for a high enough $\gamma$ the emergent symmetry restoration takes place, see Fig. 2a and $\mathrm{f}$.

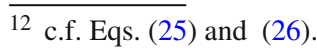

${ }^{13}$ I.e. $\mu^{2}>0$.
} 
Fig. 1 Emergent symmetry restoration, $\gamma$ dependence. The solid lines represent $\phi(t)$ for various choices of the parameter $\gamma$ in the vicinity of its critical value $\gamma_{0}$. Above each solid curve the deviation $\Delta \gamma \equiv \gamma-\gamma_{0}$ from the critical value is specified.

The dotted line represents the solution of the bare equation of motion. The parameters are chosen as follows: $\xi=-\frac{1}{12}$, $\mu=0.18$, hence $\gamma_{0} \simeq 0.294$; $\lambda=0.75, \omega=2500$; for each curve $\alpha=\left(\gamma_{0}+\Delta \gamma\right) / \omega$. The initial conditions are chosen as follows: $\phi(0)=0.6, \dot{\phi}(0)=0$

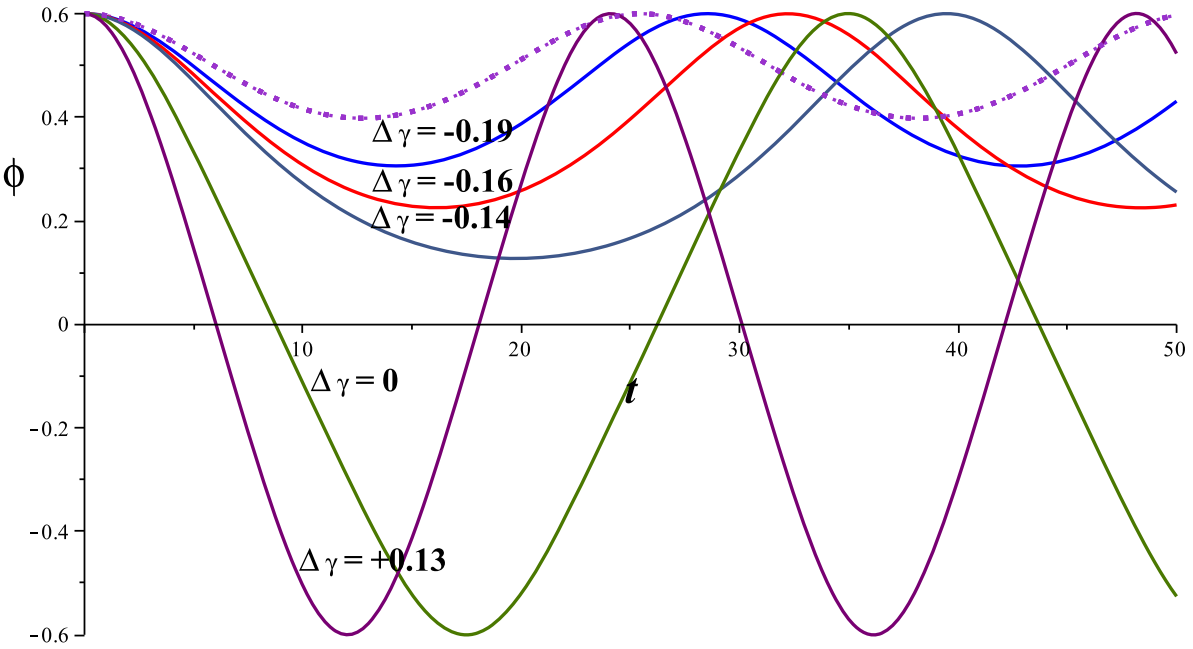

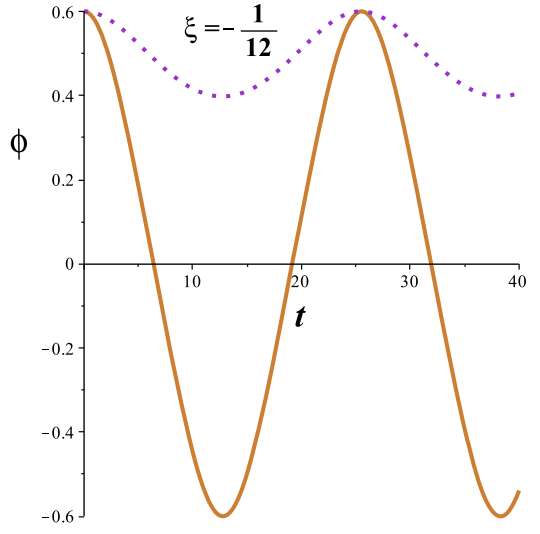

(a)

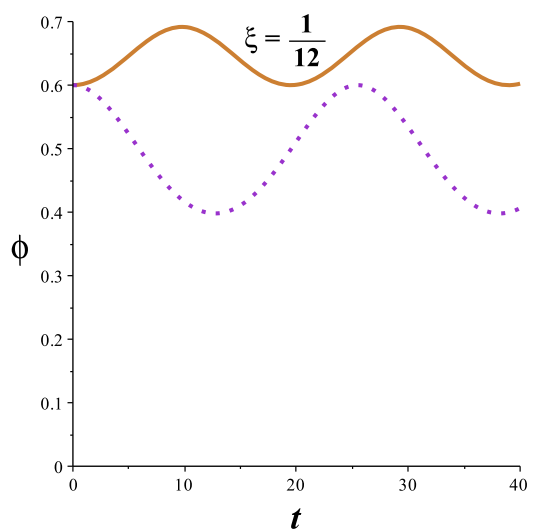

(d)

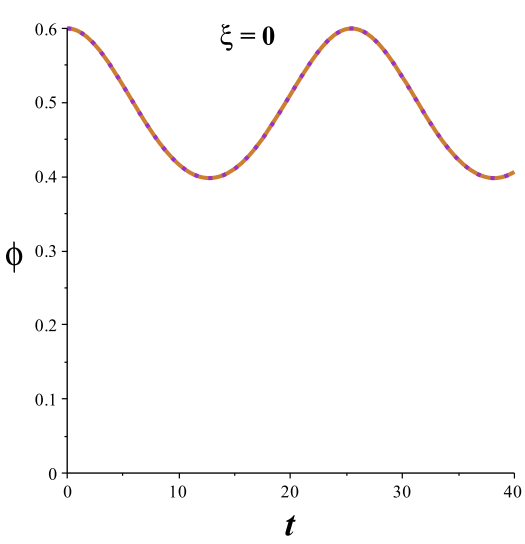

(b)

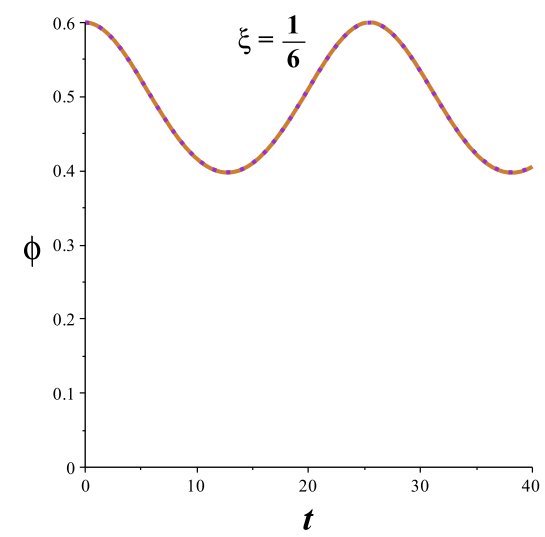

(e)

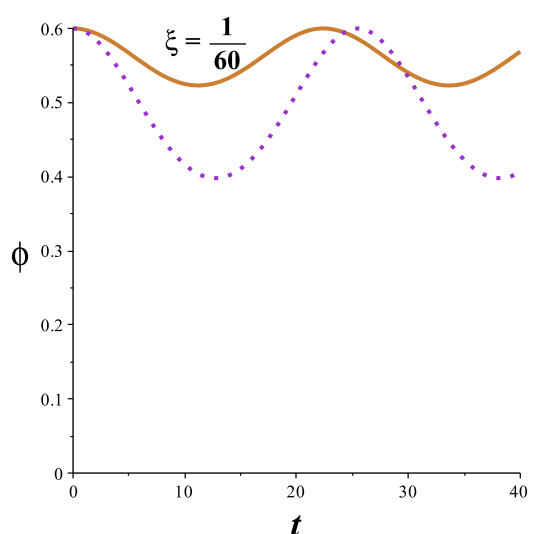

(c)

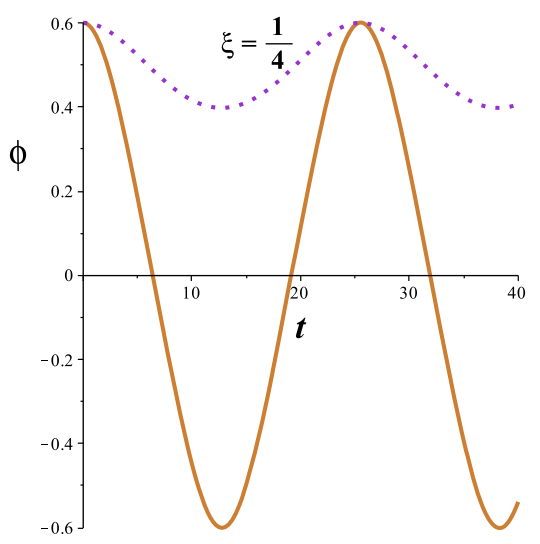

(f)
Fig. 2 Emergent symmetry restoration, $\xi$ dependence. The dotted and the solid lines represent correspondingly the bare and the perturbed solutions. The parameters are chosen as follows:

Now let us elaborate in a similar fashion the spontaneously unbroken $^{14}$ system at $\omega=0$. For various choices of the

$\overline{14}$ I.e. $\mu^{2}=-m^{2} \leq 0$. $\mu=0.18, \lambda=0.75, \gamma \equiv \omega \cdot \alpha=0.4, \omega=2500$. The initial conditions are chosen as follows: $\phi(0)=0.6, \dot{\phi}(0)=$ 0

parameter $\gamma \equiv \alpha \cdot \omega$ above and below its critical value numerical solutions of (31), (32) are presented on Fig.3. Again for small negative deviations $\Delta \gamma$ of $\gamma$ from the critical value 
Fig. 3 Emergent spontaneous symmetry breaking, $\gamma$ dependence. The solid lines represent $\phi(t)$ for various choices of the parameter $\gamma$ in the vicinity of its critical value $\gamma_{0}$. The dotted line represents the solution of the bare equation. Above each solid curve the deviation $\Delta \gamma \equiv \gamma-\gamma_{0}$ from the critical value is specified. The parameters are chosen as follows: $\xi=+\frac{1}{12}, \mu=2 \mathrm{i}$, hence $\gamma_{0} \simeq 5.657 ; \lambda=4$, $\omega=2500$; for each curve $\alpha=\left(\gamma_{0}+\Delta \gamma\right) / \omega$. The initial conditions are chosen as follows: $\phi(0)=0.2, \dot{\phi}(0)=0$

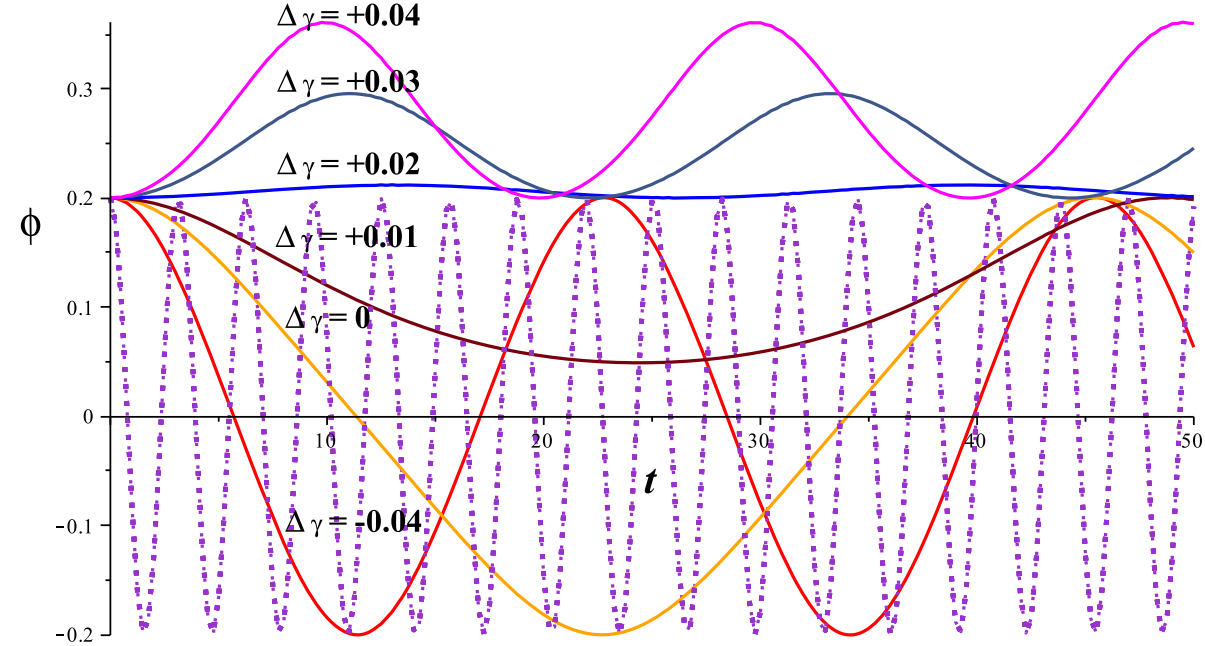

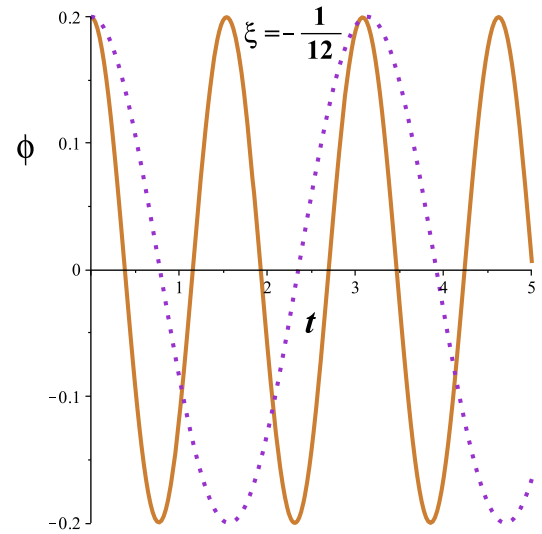

(a)

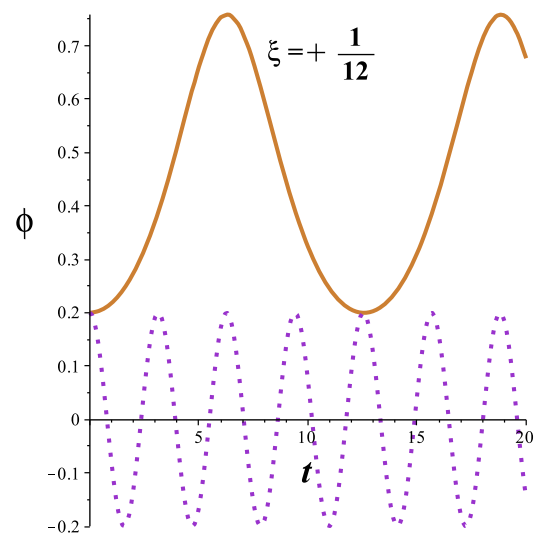

(d)

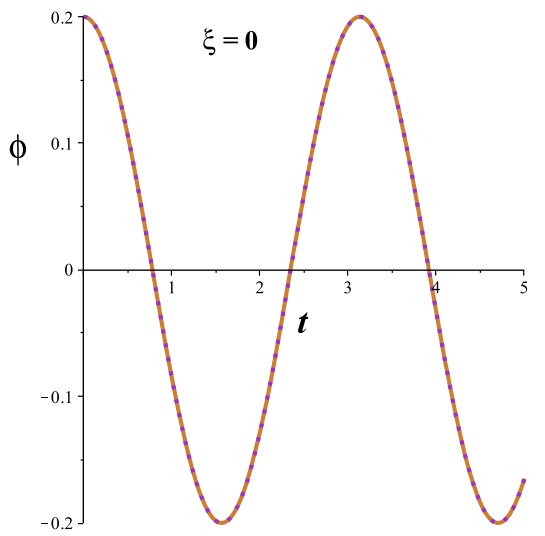

(b)

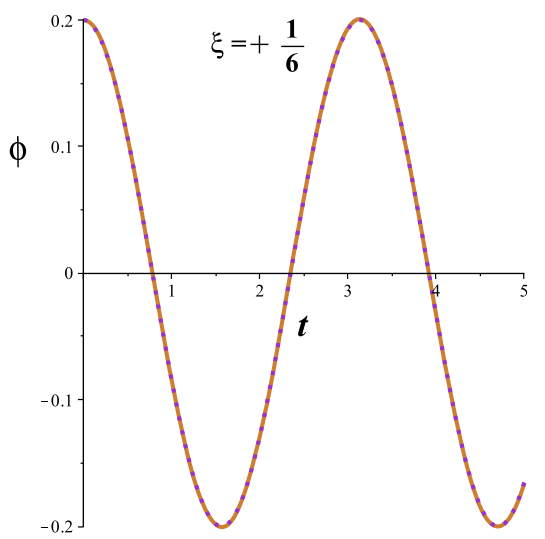

(e)

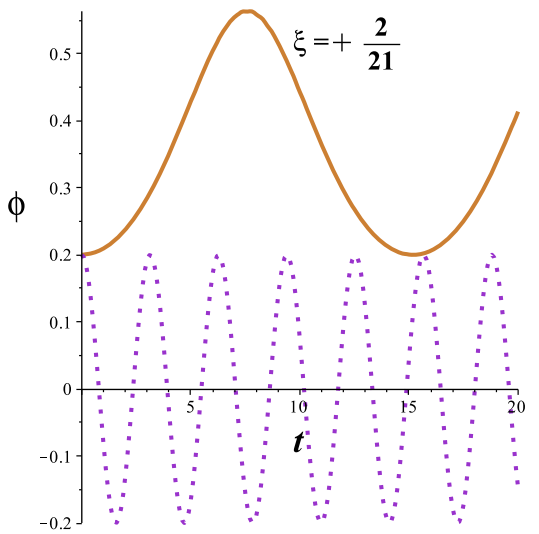

(c)

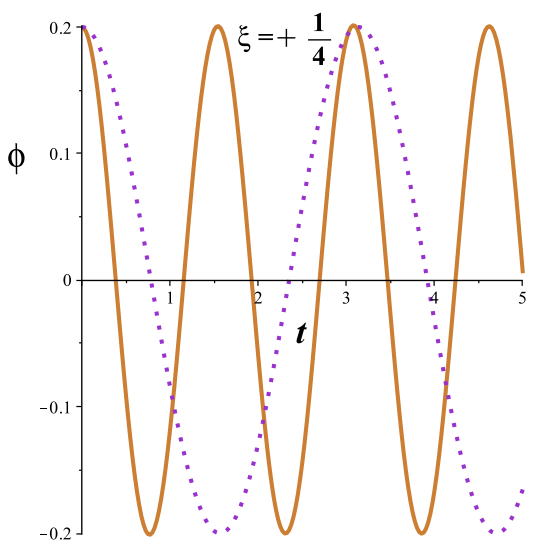

(f)
Fig. 4 Emergent spontaneous symmetry breaking, $\xi$ dependence. The dotted and the solid lines represent correspondingly the bare and the perturbed solutions. The parameters are chosen as fol- lows: $\mu=2 \mathrm{i}, \lambda=4, \gamma \equiv \omega \cdot \alpha=5.8, \omega=2500$. The initial conditions are chosen as follows: $\phi(0)=0.2, \dot{\phi}(0)=$ 0 $\gamma_{0}$ the effect of rapid oscillations results in a shift of the frequency of slow oscillations around the equilibrium point $\phi=0$. When $\Delta \gamma \geq 0$ the solutions with high precision are described by slow oscillations around the ground state at $\phi \neq 0$, which corresponds to spontaneously broken symmetry. Thus we conclude that effectively at the scales of length 


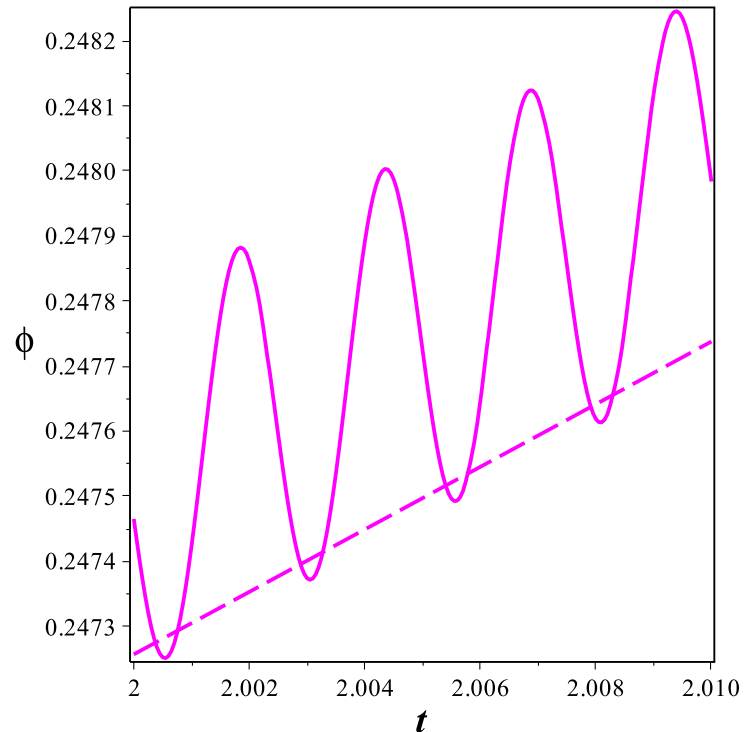

(a)

Fig. 5 Exact and approximate solutions: high resolution. The dash and the solid lines represent solutions of the effective and the exact equations respectively. The parameters are chosen as follows: $\mu=2 \mathrm{i}, \lambda=4$,

much higher than $\omega^{-1}$ the system behaves as spontaneously broken.

On Fig. 4 we illustrate the dependence on the parameter $\xi$ for this situation. At $\xi \leq 0$ and $\xi \geq 1 / 6$ the only effect is a change of the frequency of slow oscillations around the symmetric ground state $\phi=0$, see Fig. $4 \mathrm{a}$ and $\mathrm{f}$, in particular at $\xi=0$ and $\xi=1 / 6$ at the scales much higher than $\omega^{-1}$ there is no any effect at all, see Fig. $4 \mathrm{~b}$ and e. Inside the interval $0<\xi<1 / 6$ for sufficiently high values of $\gamma$ one has the phase transition - emergent spontaneous symmetry breaking, see Fig. $4 \mathrm{c}$ and $\mathrm{d}$.

In conclusion we present typical solutions of the exact (31) and the effective (32) equations at small scales, which are comparable with $\omega^{-1}$. On Fig. 5a and b the small scale behavior is presented for various time intervals. One can see that the the exact solution oscillates with the frequency $\omega$, while the difference between the two ${ }^{15}$ is indeed of the order of $\omega^{-1} \sim 10^{-3}$.

Acknowledgments The author is grateful to F. Lizzi, O. Novikov and D. Vassilevich for reading the manuscript and constructive comments. This work is supported by the FAPESP process 2015/05120-0.

Open Access This article is distributed under the terms of the Creative Commons Attribution 4.0 International License (http://creativecomm ons.org/licenses/by/4.0/), which permits unrestricted use, distribution,

\footnotetext{
15 Since deriving the effective equation we passed to the limit $\omega \rightarrow \infty$, we neglected by terms of the order of $\mathcal{O}\left(\omega^{-1}\right)$, thus the mean value of the precise solution and the effective solution may slightly differ, however this difference if of the order of $\mathcal{O}\left(\omega^{-1}\right)$.
}

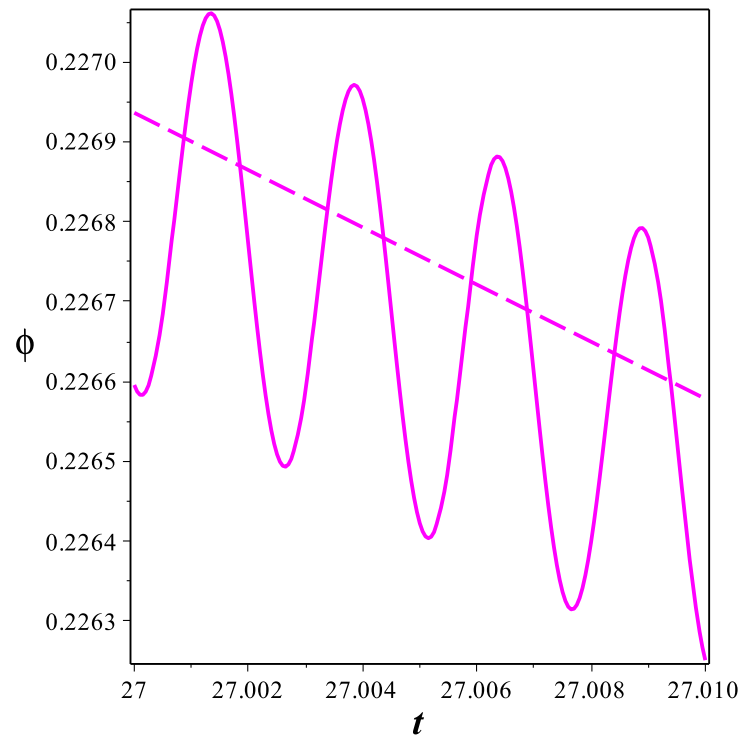

(b)

$\gamma \equiv \omega \cdot \alpha=4 \sqrt{2}+0.1, \omega=2500, \xi=1 / 12$. The initial conditions are chosen as follows: $\phi(0)=0.2, \dot{\phi}(0)=0$

and reproduction in any medium, provided you give appropriate credit to the original author(s) and the source, provide a link to the Creative Commons license, and indicate if changes were made. Funded by $\mathrm{SCOAP}^{3}$.

\section{References}

1. W.L. Power, I.C. Percival, Decoherence of quantum wavepackets due to interaction with conformal space-time fluctuations. Proc. R. Soc. Lond. A 456, 955 (2000). doi:10.1098/rspa.2000.0544. arXiv:quant-ph/9811059

2. C.H.-T. Wang, R. Bingham, J.T. Mendonca, Quantum gravitational decoherence of matter waves. Class. Quant. Grav. 23, L59 (2006). doi:10.1088/0264-9381/23/18/L01

3. R. Durrer, J. Laukenmann, The Oscillating universe: an alternative to inflation. Class. Quant. Grav. 13, 1069 (1996). doi:10.1088/ 0264-9381/13/5/021

4. H. Collins, B. Holdom, Matter in a warped and oscillating background. Phys. Rev. D 65, 124014 (2002). doi:10.1103/PhysRevD. 65.124014

5. H. Collins, B. Holdom, Decay of the cosmological constant through an oscillating metric. JHEP 0210, 052 (2002). doi: 10.1088/ 1126-6708/2002/10/052

6. H. Collins, B. Holdom, The Randall-Sundrum scenario with an extra warped dimension. Phys. Rev. D 64, 064003 (2001). doi:10. 1103/PhysRevD.64.064003

7. J.C. Hwang, Curved space quantum scalar field theory with accompanying metric fluctuations. Phys. Rev. D 48, 3544 (1993). doi: 10. 1103/PhysRevD.48.3544

8. G. Mangano, F. Lizzi, A. Porzio, Inconstant Planck's constant. Int. J. Mod. Phys. A 30(34), 1550209 (2015). doi:10.1142/ S0217751X15502097

9. M. de Cesare, F. Lizzi, M. Sakellariadou, Effective cosmological constant induced by stochastic fluctuations of Newton's constant. arXiv:1603.04170 [gr-qc] 
10. P.L. Kapitza, Dynamic stability of a pendulum when its point of suspension vibrates. Sov. Phys. JETP 21, 588-592 (1951)

11. P.L. Kapitza, Pendulum with a vibrating suspension. Usp. Fiz. Nauk 44, 7-15 (1951)
12. A. Vilenkin, L.H. Ford, Gravitational effects upon cosmological phase transitions. Phys. Rev. D 26, 1231 (1982). doi:10.1103/ PhysRevD.26.1231 